\title{
Application of the GPR and ERT methods for non-invasive examination of a flood dike
}

\author{
Tomisław Gołębiowski ${ }^{1,}$, Bogdan Piwakowski ${ }^{2}$, and Michat Ćwiklik ${ }^{1}$ \\ ${ }^{1}$ Cracow University of Technology, Faculty of Environment and Power Engineering, \\ Warszawska 24, 31-155 Cracow, Poland \\ ${ }^{2}$ Ecole Centrale de Lille, Institut d'électronique, Microélectronique \& Nanotechnologies, \\ Cité Scientifique, Avenue Henri Poincaré CS 60069, 59-652 Villeneuve d'Ascq Cedex, France
}

\begin{abstract}
As part of the NAWA-EMMAT project, geophysical surveys were carried out on selected sites in Poland, i.e. on the flood dike and in the surrounding of the concrete water dam. The goal of the surveys was the noninvasive detection of loose zones in the flood dike and fractured zones located in the subsoil of the water dam. Terrain geophysical measurements were conducted with the use of electrical and electromagnetic methods as well as applying seismic methods. Due to the limitations of the length of this paper, only selected results recorded on the flood dike using selected geophysical methods, i.e. electrical resistivity tomography (ERT) and ground penetrating radar (GPR) are presented. The presence of several higher hydraulic permeability zones in the dike was suggested by the results of the ERT method. The GPR method allowed, due to its high resolution, to identify small leakage zones in the body of the dike. An attempt at a quantitative interpretation of the geophysical data was made; for this purpose, the synthetic results obtained from numerical modelling of electrical and electromagnetic fields were applied. For proper construction of numerical models and further interpretation, the results of geotechnical sounding, geological drilling and the results of petrophysical measurements were also used.
\end{abstract}

\section{Introduction}

As part of the EMMAT (i.e. E-Mobility, sustainable MAterials and Technologies) project financed by the Polish National Agency for Academic Exchange (Polish acronym "NAWA"), geophysical surveys were carried out on two selected sites in southern Poland, i.e. on the Vistula river dike in the village of Wawrzeńczyce near Cracow and in the surrounding of concrete water dam located in the village of Wapienica near Bielsko-Biała. The aim of the geophysical surveys was to detect loose zones in the flood dike and fractured zones located in the subsoil of water dam. For this purpose, the following methods were applied: electrical resistivity tomography (ERT), electromagnetic profiling (EMP), ground penetrating radar (GPR), capacitively-coupled resistivity method (CCR) and seismic methods, in essence reflection technique and multichannel analysis of surface waves

\footnotetext{
*Corresponding author: tgolebiowski@pk.edu.pl
} 
(MASW) technique. Due to the limitations of the length of this paper, only selected results recorded on the flood dike with the use of the ERT and GPR methods have been presented.

Detection of loose zones in the flood dikes and monitoring of their development is a matter of critical interest because such zones may lead to leakage or even damage of those hydrotechnical constructions. Detection of loose zones may be carried out by the drilling of geological boreholes or by geotechnical sounding. However, both methods weaken structures, are invasive and expensive and, most importantly, deliver only information from a single point in time. One solution to the aforementioned problems might be the application of non-invasive geophysical methods which deliver continuous information from examined structures. Aside from the standard GPR and ERT techniques, additional geophysical methods, like microgravimetric technique, VLF method, radio magnetotelluric method, infrared method, self-potentials method, seismic refraction and MASW techniques have been applied over the loose zones in the flood dikes [1].

The flood dike located within the investigation site (Figs. 1A, B) was built before World War II and was reinforced in the years 1970-72. During the flood of 2010, the water channel and flood gate no. 1 were destroyed (Fig. 1C) and leakages were observed in the body of the dike (Fig. 1B). After the flood, the water channel, flood gate and dike in place no. III were reconstructed (Fig. 1D). Other parts of the dike where leakages were observed during the flood were only protected with sandbags (Fig. 1B) and no reinforcement was carried out. Therefore, the main aim of the GPR and the ERT surveys was to detect the loose zones existing in the body of the dike which may threaten the stability of this hydrotechnical construction during subsequent floods.

Based on the information from the boreholes (Fig. 1E), the body of the dike in the investigation site is built predominantly of a mixture of sand and gravel. Several measurements of the dike's height were taken along its profile and the average height being approximately $4.5 \mathrm{~m}$. In the leakage zones (Fig. 1B), geotechnical soundings (i.e. DPL Dynamic Penetration Light probing) were carried out (Fig. 1E) which indicated that the material of which the body of the dike is composed is mostly semi-compacted (the number of hits being between 5 and 25), but the compacted zones (the number of hits exceeding 25) as well as the loose zones (the number of hits being less than 5) also occur in the dike.

Two ground samples for laboratory (i.e. petrophysical) testing were taken from the boreholes (Figs. 1B, E). Both samples (named A and B) were taken from the compacted parts of the dike and they provided the background for defining the geophysical anomalies. The following petrophysical parameters were determined on the ground samples (Table 1): velocity of $P$ wave $\left(V_{p}\right)$, velocity of $S$ wave $\left(V_{s}\right)$, resistivity $(\rho)$, conductivity $(\sigma)$ and relative electrical permittivity $\left(\varepsilon_{\mathrm{r}}\right)$. It should be noted that the values of the electrical parameters (i.e. $\rho, \sigma)$ indicate that the body of the dike contains some degree of moisture.

Table 1. Petrophysical parameters of ground samples.

\begin{tabular}{|c|c|c|c|c|c|}
\hline $\begin{array}{c}\text { Mixture of sand } \\
\text { and gravel }\end{array}$ & $\begin{array}{c}\mathrm{V}_{\mathrm{p}} \\
{[\mathrm{m} / \mathrm{s}]}\end{array}$ & $\begin{array}{c}\mathrm{V}_{\mathrm{s}} \\
{[\mathrm{m} / \mathrm{s}]}\end{array}$ & $\begin{array}{c}\rho \\
{[\Omega \mathrm{m}]}\end{array}$ & $\begin{array}{c}\sigma \\
{[\mathrm{mS} / \mathrm{m}]}\end{array}$ & $\begin{array}{c}\varepsilon_{\mathrm{r}} \\
{[-]}\end{array}$ \\
\hline Sample A & 2067 & 1045 & 1028 & 0.972 & $\begin{array}{c}2.5 * \\
9^{* *}\end{array}$ \\
\hline Sample B & 1904 & 1036 & 1261 & 0.793 & $\begin{array}{c}2.6 * \\
9^{* *}\end{array}$ \\
\hline
\end{tabular}

*) values for dry grains of sand and gravel determined in laboratory conditions

**) values for a mixture of sand and gravel with some degree of moisture [2]

Geological, geotechnical and laboratory (i.e. petrophysical) information was used for proper construction of the numerical models. 

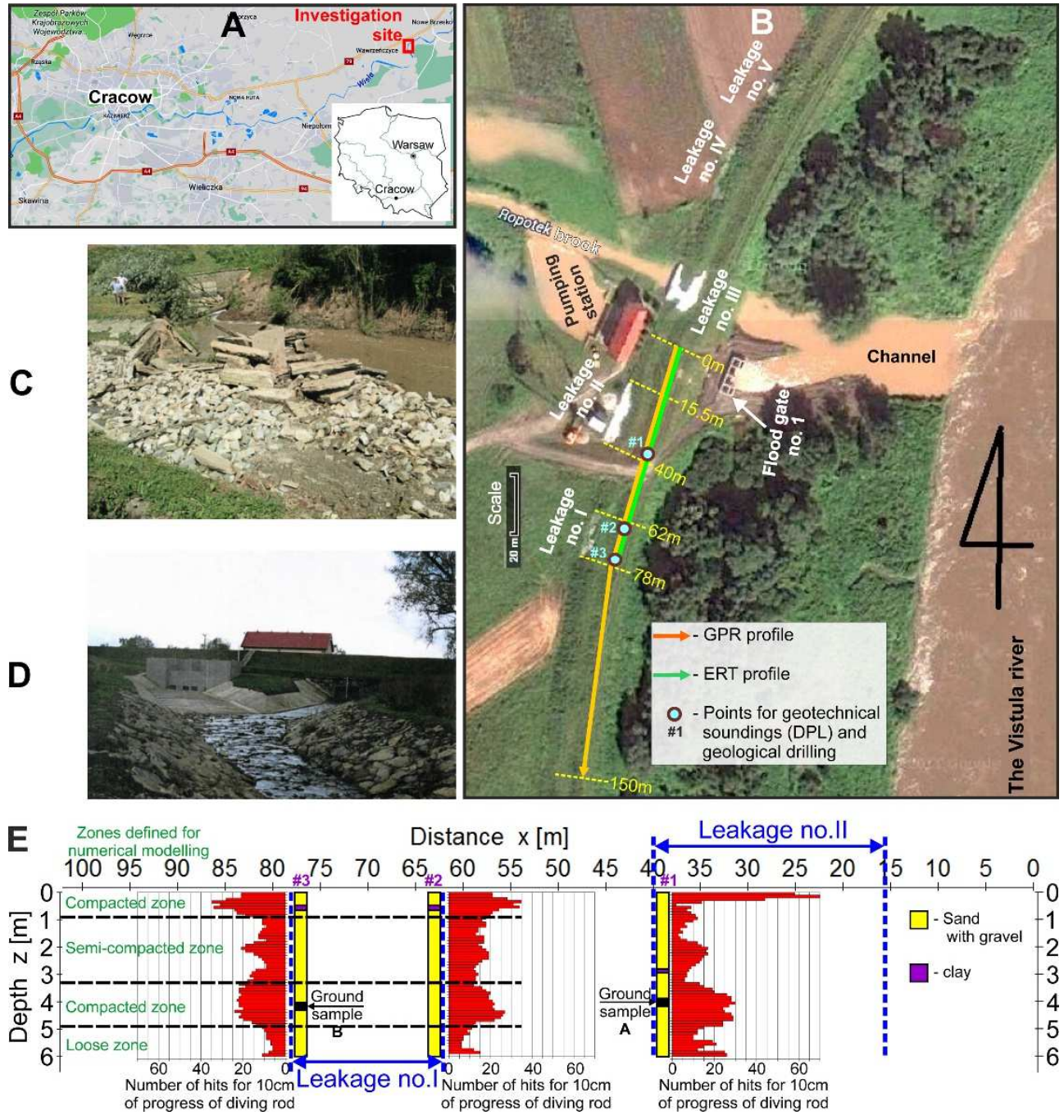

Fig. 1. A) Investigation site - the flood dike of the Vistula river near Cracow; B) Flood gate no. 1 during the flood of 2010 and the surrounding area [3]; C) Flood gate and water channel after the flood of 2010 [3]; D) Flood gate and water channel after reinforcement [3]; E) Results of geological drilling and geotechnical soundings.

\section{The GPR and ERT surveys}

Due to the limitations of this paper, the theoretical backgrounds of the GPR and ERT methods have been omitted. Such information may be found in geophysical literature $[1,2,4,5]$.

The GPR and ERT surveys were carried out along the profile of the top of the examined dike (Fig. 1B). The profile cut two regions (no. I and no. II) where leakages were observed during the flood of 2010 (Fig. 1B) where the loose zones and, consequently, the inner paths for water filtration in the body of the dike and its subsoil should occur.

The GPR terrain measurement was carried out with the use of a ProEx georadar system (manufactured by MALA - www.guidelinegeo.com) and a $200 \mathrm{MHz}$ antenna with a crosspole orientation of dipoles applied for surveys. Traces were recorded with a constant distance interval $\Delta \mathrm{x}=0.05 \mathrm{~m}$ and for improving the signal/noise ratio, 8-times stacking was applied 
during the data acquisition. The typical velocity of electromagnetic wave travelling through a dry mixture of sand and gravel is circa $0.1 \mathrm{~m} / \mathrm{ns}$. Taking into account the information from the previous section, concerning some degree of moisture of the examined dike, a reduced velocity of $0.08 \mathrm{~m} / \mathrm{hs}$ was assumed for time-depth conversion of the radargram. The recorded radargram (Fig. 2) was subjected to standard signal processing using ReflexW software (www.sandmeiergeo.de); a detailed description of the methodologies used in radargram processing may be found in geophysical literature [2].

In Fig. 2 (radargram after processing), high-amplitude anomalies were recorded along the whole profile, to the depth of circa $1.5-2 \mathrm{~m}$. This effect is caused by the reflections of electromagnetic waves from heterogeneities occurring in the mixture of sand and gravel. Additionally, this effect was enhanced by the presence of a weathered zone located in the top part of the dike. It is obvious that if during a subsequent flood water reached the top of the dike, leakages in the top part of the examined dike would appear.

Another clear anomaly in Fig. 2 is a zone with high amplitudes, between $x=20 \mathrm{~m}$ and $\mathrm{x}=44 \mathrm{~m}$ at depths $3.0 \div 3.5 \mathrm{~m}$. This anomaly appeared in the radargram over the place where leakage no. II was observed during the flood of 2010. This anomaly depicts the presence of a loose zone in the bottom part of the body of the examined dike (as highlighted before, the average height of the dike across its profile is approximately $4.5 \mathrm{~m}$ ).

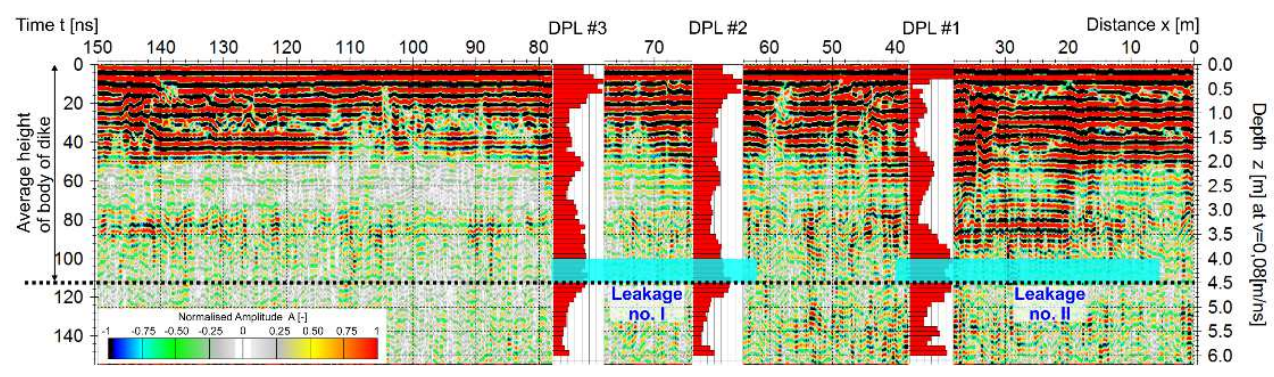

Fig. 2. Radargram after standard processing with normalised amplitude of reflections.

The GPR method did not allow for the recording of anomalies in the radargram where leakage no. I appeared during the flood. Other parts of the body of the examined dike seem to be compacted, so no additional leakages should appear during subsequent floods.

The ERT survey line running from north to south is $75 \mathrm{~m}$ long. ERT surveys were made with the basic electrode spacing of $\Delta x=2 \mathrm{~m}$. Because of a relatively good horizontal resolution and signal to noise ratio $\mathrm{S} / \mathrm{N}$, the Wenner-Schlumberger array was applied. Surveys were carried out using an ARES II resistivity meter (manufactured by GF Instruments www.gfinstruments.cz).

The obtained field data was inverted with the use of RES2DINV software (www.geotomosoft.com). A robust inversion was applied to the field data because of the lowest value of the Root Mean Square error which, in this case, equals 2.68\%. A detailed description of the inversion procedure may be found in relevant literature [5]. The inversion result is shown in Fig. 3 as interpreted resistivity cross-section.

In the geoelectrical cross-section (Fig. 3), three relatively high resistivity zones (A, B, C) are revealed and one with relatively low resistivity (D) is observed as well. Based on the results of the geotechnical soundings (Fig. 1E), the high resistivity anomalies A, B and C can be interpreted as loose and semi-compacted mixtures of gravel and sand where the central parts of the anomalies are dry (resistivities between $1000 \div 6000 \Omega \mathrm{m}$ ). Regions with resistivities between $100 \div 1000 \Omega \mathrm{m}$ can be treated as medium with some degree of moisture. It seems probable that in the case of high-water levels, during subsequent floods, the main water channels will be created in anomalies A, B and C. 
Between the two high resistivity anomalies B and C, a low resistivity area, called D, is present. Based on the resistivity value, it can be assumed that, from a geological point of view, some amount of clay should be present there. Therefore, in order to explain this phenomenon, numerical modelling will be applied to further research.

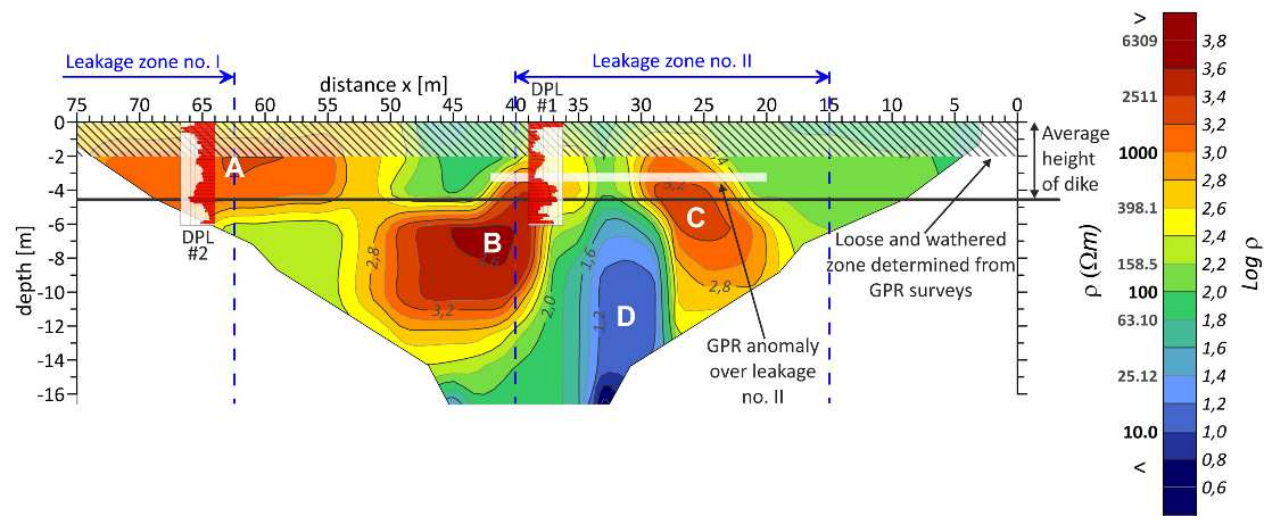

Fig. 3. The ERT field data after inversion.

Comparing the results obtained from the GPR and ERT surveys in the strip between $\mathrm{z}=0 \mathrm{~m}$ and $\mathrm{z}=2 \mathrm{~m}$ of depth, they confirm that the loose and weathered zone is more humid between $x=0 \div 24 m, x=31 \div 35 m$ and from $x=40 m$ to $x=48 m$. The GPR anomaly located over the leakage no. II correlates well with the top parts of the ERT anomalies B and C.

\section{Numerical modelling}

\subsection{Modelling of electromagnetic wave field}

The aim of the modelling of electromagnetic (em) wave field was to answer the following question: "Why, when using the GPR method, was there no anomaly recorded in the area of leakage no. I?" To answer this question, four numerical models were constructed (Figs. 4 A, C, E, G).

The most popular technique of modelling the em wave field is the FDTD (Finite Difference Time Domain) technique, and this technique is commonly used for the GPR method. Detailed information about application of the FDTD technique for radargram interpretation may be found in publication [6] and, therefore, a description of theoretical background of the FDTD method has been omitted. Numerical modelling was carried using ReflexW software (www.sandmeiergeo.de).

Based on the geological information obtained from boreholes \#2 and \#3 (Fig. 1E), a twodimensional numerical model was constructed (Fig. 4A). Vertical dimensions of the model (i.e. $7 \mathrm{~m}$ ) indicated the height of the examined dike and part of its subsoil; horizontal dimensions of models (i.e. from $\mathrm{x}=61 \mathrm{~m}$ to $\mathrm{x}=79 \mathrm{~m}$ ) were adequate to locate the region of leakage no. I (Fig. 1E).

A strip simulating air (with parameters: $\varepsilon_{\mathrm{r}}=1, \sigma=0 \mathrm{mS} / \mathrm{m}$ ) was inserted into the upper boundary of models, while on the other boundaries, the absorbing boundary condition was defined (Figs. 4A, C, E, G).

Models were discretised by a grid with dimensions $\Delta \mathrm{x}=\Delta \mathrm{z}=0.01 \mathrm{~m}$ and a time step $\Delta \mathrm{t}=0.01 \mathrm{~ns}$ was assumed for convergence and stability of the FDTD algorithm. Solving was carried out in time window $\mathrm{T}=200 \mathrm{~ns}$ according to the time window assumed during the terrain 
surveys (Fig. 2). In Fig. 2 the time window was cut at 150ns due to the lack of reflections occurring after this period.

The source of the em wave was defined as the so called "exploding reflector" and a source function with central frequency $200 \mathrm{MHz}$ was assumed based on the discretisation of the real signal taken from the radargram (i.e. from Fig. 2). In source and registration points, the crosspole geometry of the transmitter and the receiver antenna was applied.

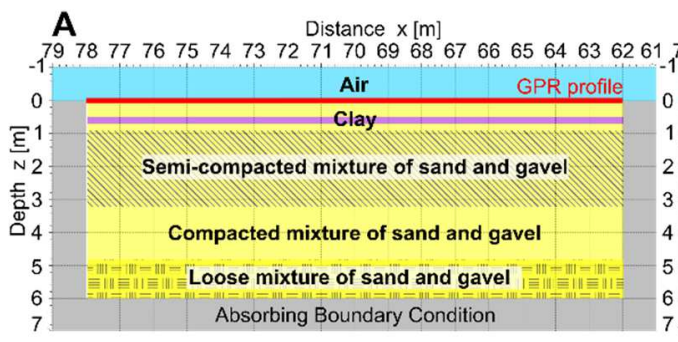

B Distance $x[\mathrm{~m}]$
C Distance $\times[\mathrm{m}]$

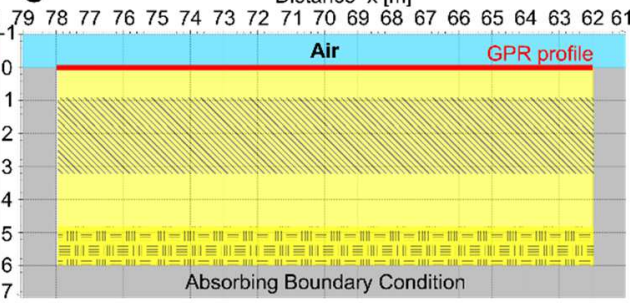

D

Distance $x[\mathrm{~m}]$

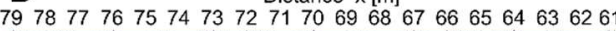

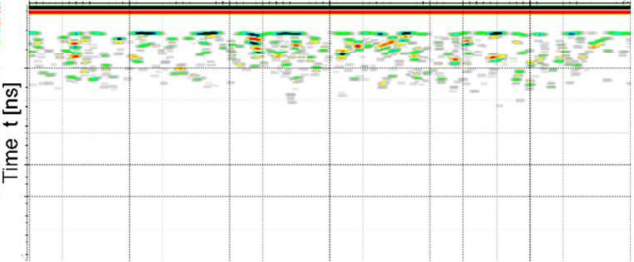

G

Distance $\times[\mathrm{m}]$
Distance $\times[\mathrm{m}]$

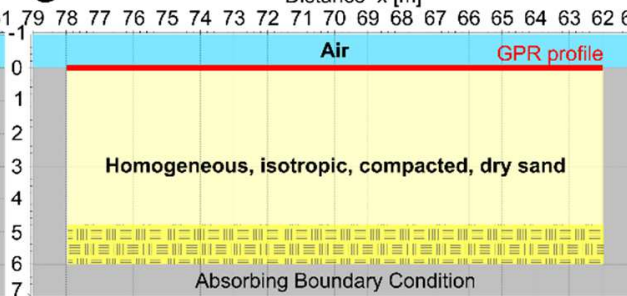

H

Distance $x[\mathrm{~m}]$

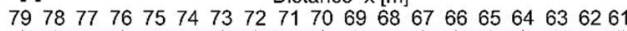

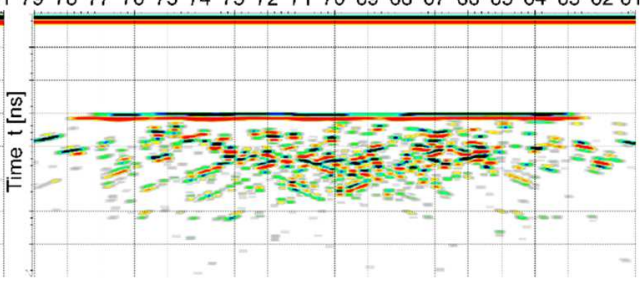

Fig. 4. Results of modelling of electromagnetic wave field: A) model constructed on the basis of geological, geotechnical and petrophysical information; B) Synthetic radargram for model A; C) Hypothetical model without layer of clay; D) Synthetic radargram form model C; E) Hypothetical model without layer of clay and semi-compacted layer; F) Synthetic radargram for model E; G) Hypothetical model with homogenous, isotropic, compacted layer; H) Synthetic radargram for model G.

Material properties of several layers within the model were defined as follow (on the base of information from Table 1 and [2]): clay $\left(\varepsilon_{\mathrm{r}}=16, \sigma=10 \mathrm{mS} / \mathrm{m}\right)$, semi-compacted mixture of sand and gravel $\left(\varepsilon_{\mathrm{r}}=2.5 \div 9, \sigma=0.1 \div 0.9 \mathrm{mS} / \mathrm{m}\right)$, compacted mixture of sand and gravel $\left(\varepsilon_{\mathrm{r}}=9\right.$, $\sigma=0.9 \mathrm{mS} / \mathrm{m})$, loose mixture of sand and gravel $\left(\varepsilon_{\mathrm{r}}=1 \div 9, \sigma=0 \div 0.9 \mathrm{mS} / \mathrm{m}\right)$; for all materials, 
constant value of relative magnetic permittivity $\mu_{\mathrm{r}}=1$ was assumed. The technique of defining material properties for the semi-compacted and loose zones has been described in paper [6].

In Fig. 4B, a synthetic radargram for modelling appropriate to the investigation site (Fig. 4A) is presented. This radargram shows that no reflections from the loose zone appear in the radargram due to the ohmic and scattering attenuation of the examined medium. Such phenomenon is observed in region of leakage no. I. Ohmic attenuation increases with increasing of conductivity of the medium, especially in clays. Scattering attenuation increases when the thickness and heterogeneity of loose (or semi-compacted) layers increase.

In Fig. 4D, a synthetic radargram for the hypothetical model (without a layer of clay Fig. 4B) is presented. In instances like this, scattering attenuation is most common. Reflections from the semi-compacted layer should probably be recorded, but an unequivocal interpretation of the thickness of such a layer would be very difficult to determine.

In Fig. 4F, a synthetic radargram for the hypothetical model (without a layer of clay and semi-compacted layer - Fig. 4E) is presented. In this scenario, only slight ohmic attenuation in the mixture of sand and gravel occurred and the recording of the reflections from the loose zone would be possible; such an instance is observed in Fig. 2 in the region of leakage no. II.

In Fig. 4H, a synthetic radargram for the hypothetical model (with a homogenous, isotropic, compacted and dry layer of sand - Fig. $4 \mathrm{G}$ ) is presented. It should be noted that the GPR detection of a loose zone would be relatively simple in such an event.

\subsection{Modelling of electrical field}

The aim of modelling of electrical field was answer the question: "What the geological layers distribution causes this kind of geoelectrical anomaly, which is observed between $x=20 \mathrm{~m}$ and $x=50 \mathrm{~m}$ of the ERT profile - Fig. 3?".

The process of electrical field modelling was carried out with the using of RES2DMOD software with the robust option constrain [7]. In the modelling process a size of the mesh was defined by the user, as follow: $\Delta \mathrm{x}=0.25 \mathrm{~m}$ and $\Delta \mathrm{z}=0.20 \mathrm{~m}$ to the depth of $9 \mathrm{~m}$ and $\Delta \mathrm{x}=0.25 \mathrm{~m}$ and $\Delta \mathrm{z}=0.50 \mathrm{~m}$ to the depth of $12 \mathrm{~m}$. The basic electrode spacing was equal $2 \mathrm{~m}, \mathrm{a}=1,3,4 \Delta \mathrm{x}$ and $n=1,2,3,4,5,6$. The Wenner-Schlumberger array was used. It should be noted that during modelling all parameters had the same values as during the field data acquisition.

The geoelectrical models (Figs. 5 A, B, C) were based on the results of DPL sounding and drilling in point \#1 (Fig. 1E). The models were also completed by the petrophysical data (Table 1) and the ERT inverted field data (Fig. 3). It is worth noting that information from point \#1 is treated like a benchmark and cannot be modified. The following values of resistivity were defined: for compacted mixture of sand and gravel assumed $\rho=1200 \Omega \mathrm{m}$ (dark violet colour in Fig. 5), for semi-compacted mixture of sand and gravel assumed $\rho=150 \Omega \mathrm{m}$ (pink colour in Fig. 5), for clay assumed $\rho=60 \Omega \mathrm{m}$ (light violet colour in Fig. 5).

The models constructed based on information from borehole and DPL sounding are layered, therefore the expected modelling results cannot reflect the field data. Thereupon, two anomalous bodies were added to the models (Fig. 5 A, B, C) with values of resistivity $\rho=1200 \Omega \mathrm{m}$ and $\rho=1000 \Omega \mathrm{m}$ which were based on the petrophysical data (Table 1 ). The shapes of bodies based on the field data inversion result (Fig. 3).

In the first variant (Fig. 5A), the bodies are intersected by the prior assumed layers. The result of modelling for model "A" was presented in Fig. 5D - the synthetic result was compared with the field data inversion result (Fig. 3) and decided that the initial model "A" should be modified to model "B" (Fig. 5B). In the next model (Fig. 5B) consider case, where the assumed layers do not intersect the anomalous bodies - the result of modelling for this case was shown in Fig. 5E. The result presented in Fig. 5E was not satisfied so third modelling was carried out for model presented in Fig. 5C. In the resistivity cross-section (Fig. 5F) it is noticeable that obtained resistivity distribution more accurately reflects the field 
data then synthetic results obtained for previous two models. Additionally, the shape and the top of the second anomalous bodies resembles in better way the field case.

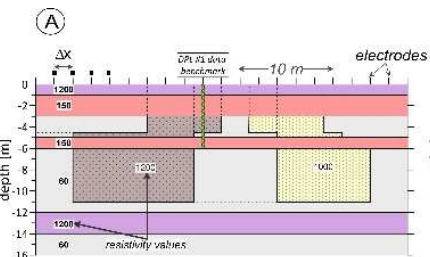

(D)

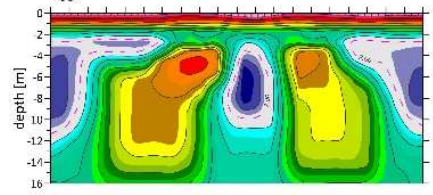

(B)

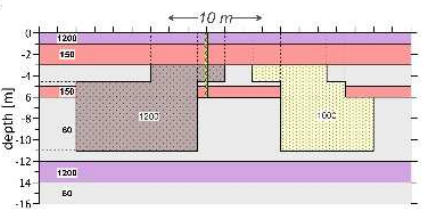

(E)

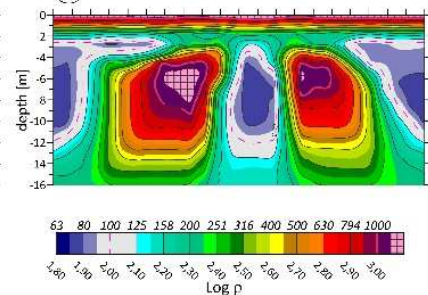

(C)

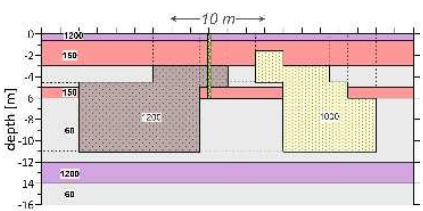

(F)

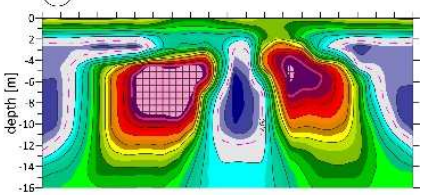

Fig. 5. Results of modelling of electrical field: A, B, C - geoelectrical models with two high resistivity bodies; D, E, F - inversion results for models A, B and C.

\section{Conclusions}

Two main issues were analysed in this paper: the first one presented the usefulness of the two selected geophysical methods, i.e. Ground Penetrating Radar (GPR) and Electrical Resistivity Tomography (ERT) for the non-invasive detection of loose zones and zones with a high permeability in a flood dike; the second examined the importance of numerical modelling for proper interpretation of recorded geophysical data. As demonstrated and discussed in this paper, only complex geophysical surveys (using a minimum of 2 complementary methods) correlated with the results of geological drillings, geotechnical soundings, laboratory (i.e. petrophysical) tests combined with the results of numerical modelling, allow for proper and unequivocal interpretation of geophysical data.

The works were financed by the grant NAWA-EMMAT, no. PPI/APM/2018/1/00027/U/001, task no. 9.

\section{References}

1. C. Fauchard, P. Mériaux, Geophysical and Geotechnical Methods for Diagnosing of Flood Protection Dikes (Cemagref Éditions, 2007)

2. A. P. Annan, Ground Penetrating Radar (Sensor \& Software Ed. 2001)

3. A. Kot, Expertise of Technical Condition of Flood Gate no. 1 Located in the Left Flood Dike of the Vistula River in the Village of Wawrzeńczyce (Aquin firm Ed. 2013)

4. J. M. Reynolds, Applied and Environmental Geophysics (Villey and Sons Ed. 2011)

5. M. H. Loke, 2-D and 3-D Electrical Imaging Surveys (Geotomo Software Ed. (2003)

6. T. Gołębiowski, Tech. Trans. 24 (2015)

7. M. H. Loke, Rapid 2D Resistivity Forward Modelling Using the Finite Difference and Finite Element Methods and 3-D Electrical Imaging Surveys (Geotomo Soft. 2002) 